PROCEEDINGS OF THE

AMERICAN MATHEMATICAL SOCIETY

Volume 127, Number 10, Pages 3013-3020

S 0002-9939(99)04343-9

Article electronically published on May 4, 1999

\title{
EXISTENCE OF ERGODIC RETRACTION FOR NONCOMMUTATIVE SEMIGROUPS IN BANACH SPACES
}

\author{
OSAMU KADA
}

(Communicated by Palle E. T. Jorgensen)

\begin{abstract}
We prove the existence of ergodic retraction for a noncommutative semigroup which is right Eberlein-weakly almost periodic in a uniformly convex Banach space.
\end{abstract}

\section{INTRODUCTION}

Let $S$ be a semitopological semigroup, i.e., a semigroup with a Hausdorff topology such that for each $t \in S$, mappings $s \mapsto s t$ and $s \mapsto t s$ from $S$ to $S$ are continuous. We denote by $\mathbb{Z}, \mathbb{Z}^{+}, \mathbb{N}, \mathbb{R}$ and $\mathbb{R}^{+}$the sets of all integers, nonnegative integers, positive integers, real numbers and nonnegative real numbers, respectively. Let $C$ be a subset of a real Banach space $E$. Then a mapping $T: C \rightarrow C$ is called nonexpansive provided $\|T x-T y\| \leq\|x-y\|$ for all $x, y \in C$. The following nonlinear ergodic theorem for nonexpansive mappings (in a Hilbert space) was established by Baillon $[1,2]$ :

Theorem 1. Let $C$ be a nonempty closed convex subset of $H$ and let $T$ be a nonexpansive mapping of $C$ into itself. If $T$ has a fixed point, then the Cesáro means $(1 / n) \sum_{k=0}^{n-1} T^{k} x$ converge weakly as $n \rightarrow \infty$ to a fixed point $y$ of $T$, and if $-C=C, 0 \in C$ and $T$ is odd, then the Cesáro means converge strongly as $n \rightarrow \infty$ to a fixed point $y$ of $T$.

In these cases, putting $y=P x$ for each $x \in C, P$ is a nonexpansive retraction of $C$ onto the set $\operatorname{Fix}(T)$ of fixed points of $T$ such that $P T^{n}=T^{n} P=P$ for all $n \in \mathbb{Z}^{+}$, and $P x \in \operatorname{cl} \operatorname{co}\left\{T^{n} x ; n \in \mathbb{Z}^{+}\right\}$for each $x \in C$, where clco $A$ is the closure of the convex hull of $A$. We call such a retraction "ergodic retraction". Takahashi [29] proved the existence of ergodic retraction for a noncommutative semigroup in a Hilbert space, and Hirano, Kido and Takahashi [12] proved it for commutative semigroups in a uniformly convex Banach space. Recently, we studied the operator $T(\mu)$ on $C$ when $\mu$ is an invariant mean on $C_{b}(S)$, which is the candidate of the ergodic retraction ([16]). But it remains open whether there exists ergodic retraction for noncommutative semigroups in Banach spaces. On the other hand, Ruess and Summers [24, 26, 27] proved strong mean ergodic theorems for Eberlein-weakly almost periodic functions over $S=\mathbb{R}^{+}$, which generalized Baillon [2] and Bruck

Received by the editors January 22, 1996 and, in revised form, July 18, 1997 and January 9, 1998.

1991 Mathematics Subject Classification. Primary 47A35, 47H09, 47H20.

(C)1999 American Mathematical Society 
$[4,5]$. Further, we generalized this for commutative semigroups and obtained some results about ergodic projections and retractions ([13]).

In this paper, we prove the existence of ergodic retraction for a noncommutative semigroup in a uniformly convex Banach space, assuming the semigroup is right Eberlein-weakly almost periodic.

\section{Preliminaries}

Throughout this paper we assume a Banach space $E$ is real. Let $S$ be a semitopological semigroup with identity, let $l^{\infty}(S)$ be the Banach space of all bounded real valued functions on $S$ with the supremum norm, and let $l^{1}(S)$ be the Banach space of all functions $f: S \rightarrow \mathbb{R}$ such that $\|f\|_{1}=\sum_{s \in S}|f(s)|<\infty$ with norm $\|\cdot\|_{1}$. Then $l^{1}(S)^{*}=l^{\infty}(S)$. We also denote by $C_{b}(S)$ the space of all bounded continuous real valued functions on $S$. Then for each $s \in S$ and $f \in l^{\infty}(S)$, we define elements $r(s) f$ and $l(s) f$ in $l^{\infty}(S)$ by $(r(s) f)(t)=f(t s)$, and $(l(s) f)(t)=f(s t)$, $t \in S$. Let $X$ be a subspace of $l^{\infty}(S)$ containing constant functions and translation invariant; i.e. for any $s \in S, r(s) X \subset X$ and $l(s) X \subset X$. Then an element $\mu$ of $X^{*}$, where $X^{*}$ is the dual space of $X$, is called a mean on $X$ if $\|\mu\|=\mu(1)=1$. As is known, $\mu$ is a mean on $X$ if and only if

$$
\inf _{s \in S} f(s) \leq \mu(f) \leq \sup _{s \in S} f(s)
$$

for each $f \in X$. A mean $\mu$ on $X$ is invariant if $\mu(r(s) f)=\mu(f)$ for all $s \in S$ and $f \in X$. A finite mean is an element of $\operatorname{co}\{\delta(s) ; s \in S\}$, where $\delta(s)(f)=f(s)$ for each $f \in l^{\infty}(S)$. Depending on time and circumstances, the values of mean $\mu$ at $f \in X$ will also be denoted by $\mu(f)$ or $\mu_{s}(f(s))$.

$S$ is called right reversible if any two closed left ideals of $S$ have nonvoid intersection. In this case, $(S, \leq)$ is a directed system when the binary relation " $\leq$ " on $S$ is defined by $s \leq t$ if and only if $\{s\} \cup \operatorname{cl}(S s) \supset\{t\} \cup \operatorname{cl}(S t), s, t \in S$.

Remark 2.1. Let $S$ be a semitopological semigroup such that the topology is normal and $C_{b}(S)$ has a right (resp. left) invariant mean. Then $S$ is right (resp. left) reversible; see Lau and Takahashi [21].

Let $C$ be a closed subset of a Banach space $E$. A mapping $V$ of $C$ into itself is called Lipschitzian if there exists $K \geq 0$ such that $\|V x-V y\| \leq K\|x-y\|$ for every $x, y \in C$. We define the Lipschitz norm of $V$ by

$$
\|V\|:=\sup \left\{\frac{\|V x-V y\|}{\|x-y\|} ; x, y \in C, x \neq y\right\} .
$$

If $\|V\| \leq 1$, then $V$ is called nonexpansive. We denote by $\operatorname{Lip}(C), \operatorname{Cont}(C), \operatorname{Aff}(C)$ and $L(E)$, the semitopological semigroup of all Lipschitzian self-mappings of $C$, the semitopological semigroup of all nonexpansive self-mappings of $C$, the semitopological semigroup of all affine self-mappings of $C$ and the semitopological semigroup of all bounded linear mappings of $E$, under composition and pointwise convergence topology, respectively. Let $T: S \rightarrow \operatorname{Lip}(C)$, Cont $(C)$ or $L(E)$ be a representation (resp. anti-representation), i.e., $T(s+t)=T(s) T(t)$ (resp. $T(s t)=T(t) T(s)$ ) for every $s, t \in S$, and $T(\cdot) x$ is continuous for every $x \in C$ or $E$. We denote by $\operatorname{Fix}(T)$ the set of common fixed points of $T(s), s \in S$, and by $O(x)=\{T(s) x ; s \in S\}$ the orbit from $x \in C$. We also denote by $B(E)=\{x \in E ;\|x\| \leq 1\}$ the unit ball of $E$. For two Banach spaces $E$ and $F, L(E, F)$ is the set of all bounded linear mappings from $E$ to $F$. 
First we give a lemma.

Lemma 2.2. Let $S$ be a semitopological semigroup such that there exists a left invariant mean $\mu$ on $C_{b}(S)$. Let $C$ be a closed convex subset of a Banach space $E$ and let $D$ be a bounded subset of $C$. Let $T: S \rightarrow \operatorname{Cont}(C)$ be a representation such that $\left\{T(s) x_{0} ; s \in S\right\}$ is bounded for some $x_{0} \in C$. Then there exists a bounded closed convex subset $M$ of $C$, which is $T(s)$-invariant for every $s \in S$ and includes $K:=\{T(s) x ; s \in S, x \in D\}$.

Proof. Put $a=\sup _{s \in S}\left\|T(s) x_{0}\right\|, b=\sup _{x \in D}\|x\|$, and define the set

$$
M:=\left\{z \in C ; \mu_{s}\left\|T(s) x_{0}-z\right\| \leq a+b\right\} .
$$

Then we can easily see that $M$ is as in the assertion.

Let $S$ be a topological space, let $E$ be a Banach space $E$ and let $C_{b}(S, E)$ be the Banach space of all bounded continuous mappings from $S$ to $E$ with supremum norm, and let $C_{C}(S, E)$ be the set of all elements $f \in C_{b}(S, E)$ such that $f(S):=$ $\{f(s) ; s \in S\}$ is relatively weakly compact. Then $C_{C}(S, E)$ is a linear subspace of $C_{b}(S, E)$. For any mean $\mu$ on $C_{b}(S)$, we define a "vector valued mean" $\tau(\mu)$, homomorphically; i.e., $\tau(\mu)$ is an element of $L\left(C_{C}(S, E), E\right)$ such that $\tau(\mu) x=x$ for all constant functions $x \in E$, and $\|\tau(\mu)\|=1$. More generally, we give definitions as follows; see [18].

Definition 2.3. Let $S$ be a topological space and let $E$ be a Banach space. For $\mu \in C_{b}(S)^{*}$ and $f \in C_{C}(S, E)$, let $x_{\mu, f}^{* *}: x^{*} \mapsto \mu_{s}\left\langle f(s), x^{*}\right\rangle$ be an element of $E^{* *}$. Then $x_{\mu, f}^{* *} \in E$. We define an element $\tau=\tau^{E} \in L\left(C_{b}(S)^{*}, L\left(C_{C}(S, E), E\right)\right)$ by $\tau(\mu) f=x_{\mu, f}^{* *}$.

Remark 2.4. The following holds; see [18]. (i) If $\mu$ is a mean on $C_{b}(S)$, then $\tau(\mu) f \in$ $\operatorname{cl} \operatorname{co}\{f(s) ; s \in S\}$; (ii) $\tau$ is injective and $\|\tau\| \leq 1$; (iii) $\tau(\mu) x=\mu(1) x$ for all $x \in E$, and if $\mu$ is a mean on $C_{b}(S)$, then $\|\tau(\mu)\|=1$; (iv) $\tau$ maps the point evaluation $\delta(s)$ to the point evaluation $\varepsilon(s), s \in S$, where $\varepsilon(s) f=f(s)$ for $f \in C_{C}(S, E)$; (v) $\tau\left(r(s)^{*} \mu\right)=r(s)^{*} \tau(\mu)$.

Let $T: S \rightarrow \operatorname{Lip}(C)$ be a representation such that $T(\cdot) x \in C_{C}(S, E)$ for some $x \in C$. Then we shall denote $\tau(\mu)(T(\cdot) x)$ by $T(\mu) x$, which is an element of $E$.

Remark 2.5. From (ii) and (iii), for any $\left\{a_{i}\right\} \subset \mathbb{R},\left\{s_{i}\right\} \subset S, f \in C_{C}(S, E)$, we get $\tau\left(\sum_{i=1}^{n} a_{i} \delta\left(s_{i}\right)\right) f=\sum_{i=1}^{n} a_{i} \tau\left(\delta\left(s_{i}\right)\right) f=\sum_{i=1}^{n} a_{i} \varepsilon\left(s_{i}\right) f=\sum_{i=1}^{n} a_{i} f\left(s_{i}\right)$.

We give two lemmas.

Lemma 2.6. Let $f \in C_{C}(S, E)$ and let $\mu$ be a left (resp. right) invariant mean on $C_{b}(S)$. Then $\tau(\mu) f \in \bigcap_{s \in S} \operatorname{cl} \operatorname{co}\{f(s t) ; t \in S\}\left(\right.$ resp. $\tau(\mu) f \in \bigcap_{s \in S} \operatorname{cl} \operatorname{co}\{f(t s) ; t \in$ $S\})$. If $\mu$ is an invariant mean on $C_{b}(S), \tau(\mu) f \in \bigcap_{s, q \in S} \operatorname{cl} \operatorname{co}\{f(s t q) ; t \in S\}$.

Proof. For any $s \in S$, considering $l(s) f$ for $f$ in Remark 2.4(i), we have $\tau(\mu) f=$ $\tau(\mu)(l(s) f) \in \operatorname{cl} \operatorname{co}\{(l(s) f)(t) ; t \in S\}=\operatorname{cl} \operatorname{co}\{f(s t) ; t \in S\}$. So, the first assertion follows. The other assertions are proved similarly.

Lemma 2.7. Let $\mu$ be a right invariant mean on $C_{b}(S)$ and let $\lambda$ be a finite mean. Putting $f(t)=T\left(l(t)^{*} \lambda\right) x$ for every $t \in S$ and $x \in E$, we have $T(\mu) x=\tau(\mu) f$. 
Proof. Let $\lambda=\sum_{i=1}^{n} a_{i} \delta\left(s_{i}\right)$. Then by Remark 2.5, $T\left(l(t)^{*} \lambda\right) x=\sum_{i=1}^{n} a_{i} T\left(t s_{i}\right) x$, hence

$$
\begin{aligned}
\tau(\mu) f & =\tau(\mu)\left(T\left(l(\cdot)^{*} \lambda\right) x\right)=\tau(\mu)\left(\sum_{i=1}^{n} a_{i} T\left(\cdot s_{i}\right) x\right) \\
& =\sum_{i=1}^{n} a_{i} \tau(\mu)\left(T\left(\cdot s_{i}\right) x\right)=\sum_{i=1}^{n} a_{i} \tau(\mu)(T(\cdot) x) \\
& =\tau(\mu)(T(\cdot) x)=T(\mu) x .
\end{aligned}
$$

Therefore $T(\mu) x=\tau(\mu) f$.

\section{EXISTENCE OF ERGODIC RETRACTION}

In this section, we prove that there exists the ergodic retraction for a noncommutative semigroup which is right Eberlein-weakly almost periodic. First, we give the definition of vector valued weakly almost periodic functions; see Ruess and Summers [24].

Definition 3.1. Let $S$ be a semitopological semigroup and let $E$ be a Banach space. We say a function $f \in C_{b}(S, E)$ is right (resp. left) Eberlein-weakly almost periodic if $\{r(s) f ; s \in S\}$ (resp. $\{l(s) f ; s \in S\}$ ) is a relatively weakly compact subset of $C_{b}(S, E)$. If $f \in C_{b}(S, E)$ is right and left Eberlein-weakly almost periodic, then we say $f$ is Eberlein-weakly almost periodic. We denote by $W R(S, E)$ (resp. $W L(S, E), W(S, E)$ ) the space of all of right Eberlein- (resp. left Eberlein-, Eberlein-) weakly almost periodic functions. Let $R U C(S, E)$ be the set of all right bounded uniformly continuous functions from $S$ to $E$, i.e., the set of all $f \in C_{b}(S, E)$ such that the map $s \mapsto r(s) f$ from $S$ to $C_{b}(S, E)$ is continuous. Let $C$ be a closed subset of $E$. We say a representation $T: S \rightarrow \operatorname{Cont}(C)$ is right Eberlein- (resp. left Eberlein-, Eberlein-) weakly almost periodic on a subset $D$ of $C$ if for any $x \in D, T(\cdot) x \in W R(S, E)(\operatorname{resp} . W L(S, E), W(S, E))$.

Proposition 3.2. (a) $W R(S, E), W L(S, E)$ and $W(S, E)$ are closed translation invariant linear subspaces of $C_{b}(S, E)$, and (b) $R U C(S, E)$ is a closed left translation invariant linear subspace of $C_{b}(S, E)$.

Proof. (a) (i) Closeness of $W R(S, E), W L(S, E)$ and $W(S, E)$ : this is proved just as in the proof of Berglund, Junghenn and Milnes [3, Theorem 4.2.5].

(ii) Translation invariance of $W R(S, E), W L(S, E)$ and $W(S, E)$ : For $f \in$ $W R(S, E)$ and $t \in S$, since $\{r(s) r(t) f ; s \in S\} \subset\{r(s) f ; s \in S\}$, we see that $r(t) f \in W R(S, E)$. So, $W R(S, E)$ is right translation invariant.

Let $f \in W R(S, E)$ and $t \in S$. Since $\operatorname{cl}\{r(s) l(t) f ; s \in S\}=\operatorname{cl}\{l(t) r(s) f ; s \in S\}=$ $l(t) \operatorname{cl}\{r(s) f ; s \in S\}$, and $l(t)$ is weak to weak continuous, $\operatorname{cl}\{r(s) l(t) f ; s \in S\}$ is weakly compact. So, we have $l(t) f \in W R(S, E)$, and $W R(S, E)$ is left translation invariant. Similarly, we see $W L(S, E)$ and $W(S, E)$ are translation invariant.

(b) Closeness of $R U C(S, E)$ : Let $f \in \operatorname{cl} R U C(S, E)$, and let $\left\{f_{\alpha}\right\} \subset R U C(S, E)$ be such that $f_{\alpha} \rightarrow f$. We show $f \in R U C(S, E)$. For this it is sufficient to show the following: let $\left\{s_{\beta}\right\}$ be a net of $S$ such that $s_{\beta} \rightarrow s$. Then $r\left(s_{\beta}\right) f \rightarrow r(s) f$. For any $\varepsilon>0$, there exists $\alpha=\alpha(\varepsilon)$ such that

$$
\left\|f-f_{\alpha}\right\| \leq \varepsilon .
$$


Then there exists $\beta_{0}=b_{0}(\alpha)$ such that for any $\beta \geq \beta_{0}$,

$$
\left\|r\left(s_{\beta}\right) f_{\alpha}-r(s) f_{\alpha}\right\| \leq \varepsilon
$$

Then we have

$$
\begin{aligned}
\left\|r\left(s_{\beta}\right) f-r(s) f\right\| & \leq\left\|r\left(s_{\beta}\right) f-r\left(s_{\beta}\right) f_{\alpha}\right\|+\left\|r\left(s_{\beta}\right) f_{\alpha}-r(s) f_{\alpha}\right\|+\left\|r(s) f_{\alpha}-r(s) f\right\| \\
& \leq \varepsilon+2\left\|f-f_{\alpha}\right\| \leq 3 \varepsilon .
\end{aligned}
$$

This implies $r\left(s_{\beta}\right) f \rightarrow r(s) f$.

(ii) Left translation invariance of $R U C(S, E)$ : Let $f \in R U C(S, E)$ and $q \in S$. Then we have

$$
\begin{aligned}
\|r(s) l(q) f-r(t) l(q) f\| & =\|l(q) r(s) f-l(q) r(t) f\| \\
& \leq\|r(s) f-r(t) f\|,
\end{aligned}
$$

so that $l(q) f \in R U C(S, E)$.

Remark 3.3. Let $C$ be a closed convex subset of a uniformly convex Banach space $E$ and let $T: S \rightarrow \operatorname{Cont}(C)$ be a representation such that $\operatorname{Fix}(T) \neq \varnothing$. Then we know that when $S=\left(\mathbb{Z}^{+}\right)^{d}$ or $\left(\mathbb{R}^{+}\right)^{d}, d \in \mathbb{N}$, if the representation $T$ is strongly asymptotically isometric on a subset $D$ of $C$, then $T$ is Eberlein-weakly almost periodic on $D$. Here $T$ is called strongly asymptotically isometric on $D$ if the following hold: Let $\|\cdot\|$ be the restriction to $S$ of any norm on $(\mathbb{R})^{d}$. For any $x, y \in D, \lim _{\|s\| \rightarrow \infty}\|T(s+h) x-T(s) y\|$ exists uniformly over $h \in S$ (see $[13,24]$ ).

As in the proof of Ruess and Summers [27, Proposition 2.1], we have the following lemma.

Lemma 3.4. $f \in W R(S, E)$ (resp. $W L(S, E))$ if and only if $\left\{\left\langle f(t \cdot), x^{*}\right\rangle ; t \in S, x^{*} \in\right.$ $\left.B\left(E^{*}\right)\right\}$ (resp. $\left\{\left\langle f(\cdot t), x^{*}\right\rangle ; t \in S, x^{*} \in B\left(E^{*}\right)\right\}$ ) is relatively weakly compact in $C_{b}(S)$.

Proof. By Milnes [22], $f \in W R(S, E)$ if and only if for any sequences $\left\{\left(t_{m}, x_{m}^{*}\right)\right\}$ in $S \times B\left(E^{*}\right)$ and $\left\{s_{n}\right\}$ in $S$,

$$
\lim _{n} \lim _{m}\left\langle f\left(t_{m} s_{n}\right), x_{m}^{*}\right\rangle=\lim _{m} \lim _{n}\left\langle f\left(t_{m} s_{n}\right), x_{m}^{*}\right\rangle,
$$

whenever both iterated limits exists. From this, the assertion follows. The second assertion is proved similarly.

Let $C$ be a closed convex subset of a Banach space $E$, and let $T$ be a nonexpansive mapping on $C$ and $\varepsilon>0$. Then we define

$$
F_{\varepsilon}(T)=\{x \in C ;\|x-T x\| \leq \varepsilon\} .
$$

The following is Bruck's result [5].

Lemma 3.5. Let $C$ be a bounded closed convex subset of a uniformly convex Banach space $E$. Then for any $\varepsilon>0$ and any nonexpansive mapping $T$ on $C$, there exists $\delta>0$ such that

$$
\operatorname{clco} F_{\delta}(T) \subset F_{\varepsilon}(T) .
$$

The following lemma is a noncommutative version of a result of Hirano, Kido and Takahashi [11]; see [16]. 
Lemma 3.6. Let $C$ be a closed subset of a Banach space $E$, let $S$ be a right reversible semitopological semigroup, and let $T: S \rightarrow \operatorname{Cont}(C)$ be a representation. Assume $\operatorname{Fix}(T) \neq \varnothing$. Then for any $x \in C$ and any finite mean $\lambda \in \operatorname{co}\{\delta(s) ; s \in S\}$,

$$
\lim _{t}\left\|T(s) T\left(l(t)^{*} \lambda\right) x-T\left(l(s t)^{*} \lambda\right) x\right\|=0 \quad \text { uniformly over } s \in S .
$$

Let $\langle F, G\rangle$ be a duality of vector spaces. We do not assume it is separated. Let $\sigma(F, G)$ be the weak topology on $F$ generated by $G$, and let $\tau(F, G)$ be the Mackey topology on $F$, i.e., the topology of uniform convergence on all $\sigma(G, F)$-compact convex circled subsets of $G$.

Lemma 3.7. Let $X$ be a translation invariant closed subspace of $l^{\infty}(S)$ containing constants, and assume that $X$ has a left invariant mean $\mu$. Then there exist nets $\left\{\phi_{\alpha}\right\}$ and $\left\{\varphi_{\alpha}\right\}$ of finite means such that for any $s \in S, l(s)^{*} \phi_{\alpha}-\phi_{\alpha}$ and $l(s)^{*} \varphi_{\alpha}-\mu$ converge to 0 in the $\tau\left(l^{1}(S), X\right)$ topology and $\tau\left(X^{*}, X\right)$ topology, respectively.

Proof. The first assertion is proved in [16]. The second assertion is proved similarly. Indeed, by the Mackey-Arens theorem, the continuous dual of $\left(X^{*}, \tau\left(X^{*}, X\right)\right)$ is also $X$. Let $E$ be the product $\left(X^{*}, \tau\left(X^{*}, X\right)\right)^{S}$. Let $\Phi$ be the set of finite means and define a map $T: \Phi \rightarrow E$ given by

$$
T(\phi)(s)=l(s)^{*} \phi-\mu, \quad \phi \in \Phi, s \in S .
$$

Then $T(\Phi)$ is a convex subset of $E$. There exists a net $\left\{\lambda_{\alpha}\right\} \subset \Phi$ such that $\left\langle f, l(s)^{*} \lambda_{\alpha}-\mu\right\rangle \rightarrow 0$ for each $s \in S$ and $f \in X$. Hence $T\left(\lambda_{\alpha}\right) \rightarrow 0$ in the weak topology of $E$, i.e., $0 \in$ the weak closure of $T(\Phi)$. Hence by Mazur's theorem, $0 \in$ the strong closure of $T(\Phi)$, that is, there exists a net $\left\{\varphi_{\alpha}\right\}$ of finite means such that $l(s)^{*} \varphi_{\alpha}-\mu$ converges to 0 in the $\tau\left(X^{*}, X\right)$ topology for each $s \in S$.

Now we can prove our main theorem on the existence of ergodic retraction for a noncommutative semigroup which is right Eberlein-weakly almost periodic in a uniformly convex Banach space.

Theorem 3.8. Let $S$ be a normal semitopological semigroup such that there exists a left invariant mean on $C_{b}(S)$. Let $C$ be a closed convex subset of a uniformly convex Banach space $E$, and let $T: S \rightarrow \operatorname{Cont}(C)$ be a right Eberlein-weakly almost periodic representation on a subset $D$ of $C$. Assume $\operatorname{Fix}(T) \neq \varnothing$. Then for each right invariant mean $\mu$ on $C_{b}(S)$, if it exists, $T(\mu)$ is a nonexpansive retraction from $D$ onto $\operatorname{Fix}(T)$ such that $T(\mu) T(s)=T(s) T(\mu)=T(\mu)$ for each $s \in S$ and $T(\mu) x \in \operatorname{clco} O(x)$ for each $x \in C$.

Proof. By Lemma 2.2, we may assume $C$ is bounded. Fix any $s \in S$ and $\varepsilon>0$. By Lemma 3.5, there exists $\delta>0$ such that

$$
\operatorname{clco} F_{\delta}(T(s)) \subset F_{\varepsilon}(T(s)) .
$$

Let $\lambda$ be a left invariant mean. Since $T(\cdot) x \in W R(S, E)$, by Lemma 3.4 and Krein's theorem we have that the convex, circled hull of $\left\{\left\langle T(t \cdot) x, x^{*}\right\rangle \mid ; t \in S, x^{*} \in B\left(E^{*}\right)\right\}$ is relatively weakly compact. So, by Lemma 3.7 there exists a finite mean $\lambda_{0}$ such that the following holds:

$$
\sup \left\{\left|\left(\lambda_{0}-\lambda\right)\left\langle T(t \cdot) x, x^{*}\right\rangle\right| ; t \in S, x^{*} \in B\left(E^{*}\right)\right\} \leq \delta / 4 .
$$


Then, for any $t \in S$,

$$
\begin{aligned}
\left\|T\left(l(s t)^{*} \lambda_{0}\right) x-T\left(l(t)^{*} \lambda_{0}\right) x\right\| \\
=\left\|\tau\left(l(s t)^{*} \lambda_{0}-l(t)^{*} \lambda_{0}\right)(T(\cdot) x)\right\| \\
\leq\left\|\tau\left(l(s t)^{*} \lambda_{0}-\lambda\right)(T(\cdot) x)\right\|+\left\|\tau\left(l(t)^{*} \lambda_{0}-\lambda\right)(T(\cdot) x)\right\| \\
=\sup \left\{\left(\lambda_{0}-\lambda\right)\left\langle T(s t \cdot) x, x^{*}\right\rangle ; x^{*} \in B\left(E^{*}\right)\right\} \\
\quad+\sup \left\{\left(\lambda_{0}-\lambda\right)\left\langle T(t \cdot) x, x^{*}\right\rangle ; x^{*} \in B\left(E^{*}\right)\right\} \\
\leq \delta / 4+\delta / 4=\delta / 2 .
\end{aligned}
$$

By Remark 2.1 and Lemma 3.6, there exists $t_{0} \in S$ such that

$$
\left\|T(s) T\left(l(t)^{*} \lambda_{0}\right) x-T\left(l(s t)^{*} \lambda_{0}\right) x\right\| \leq \delta / 2 \text { for all } t \geq t_{0} .
$$

Put $f(t)=T\left(l(t)^{*} \lambda_{0}\right) x$. Then for any $t \geq t_{0}$,

$$
\begin{aligned}
& \| T(s) \\
& \left.\quad=\| T(s) T(l(t))^{*} \lambda_{0}\right) x-T\left(l(t)^{*} \lambda_{0}\right) x \| \\
& \quad \leq\left\|T(s) T\left(l(t)^{*} \lambda_{0}\right) x-T\left(l(s t)^{*} \lambda_{0}\right) x\right\|+\left\|T\left(l(s t)^{*} \lambda_{0}\right) x-T\left(l(t)^{*} \lambda_{0}\right) x\right\| \\
& \quad \leq \delta / 2+\delta / 2=\delta .
\end{aligned}
$$

So, we have

$$
f(t) \in F_{\delta}(T(s)) \text { for all } t \geq t_{0} .
$$

By Lemma 2.7, Lemma 2.6, (3.2) and (3.1), we have $T(\mu) x=\tau(\mu) f \in \operatorname{cl} \operatorname{co}\{f(t) ; t \geq$ $\left.t_{0}\right\} \subset \operatorname{clco} F_{\delta}(T(s)) \subset F_{\varepsilon}(T(s))$. Since $\varepsilon>0$ and $s \in S$ are arbitrary, we obtain $T(\mu) x \in \operatorname{Fix}(T)$. So, for any $s \in S, T(s) T(\mu)=T(\mu)$. Since $\mu$ is right invariant, we have, using Remark 2.4,

$$
\begin{aligned}
T(\mu) T(s) x & =\tau(\mu)(T(\cdot) T(s) x)=\tau(\mu)(T(\cdot s) x) \\
& =\tau\left(r(s)^{*} \mu\right)(T(\cdot) x)=\tau(\mu)(T(\cdot) x) \\
& =T(\mu) x,
\end{aligned}
$$

and hence $T(\mu) T(s)=T(\mu)$ for every $s \in S$. Since $T(\mu)^{2} x=\tau(\mu)(T(\cdot) T(\mu) x)=$ $\tau(\mu) T(\mu) x=T(\mu) x$, we obtain $T(\mu)^{2}=T(\mu)$, which means $T(\mu)$ is a retraction. From Lemma 2.6, we have $T(\mu) x \in \operatorname{cl} \operatorname{co} O(x)$.

$$
\begin{aligned}
\|T(\mu) x-T(\mu) y\| & =\|\tau(\mu)(T(\cdot) x-T(\cdot) y)\| \leq\|T(\cdot) x-T(\cdot) y\| \\
& \leq\|x-y\|,
\end{aligned}
$$

so that $T(\mu)$ is nonexpansive. This completes the proof.

\section{REFERENCES}

[1] J. B. Baillon, Un théorèm de type ergodic pour les contractions non linéaires dans un espace de Hilbert, C. R. Acad. Sci. Paris Sér. A-B 280 (1975), 1511-1514. MR 51:11205

[2] - Quelques propiétés de convergence asymptotic pour les semigroupes de contractions impaires, C. R. Acad. Sci. Paris Sér. A-B 283 (1976), 75-78. MR 55:1153

[3] J. F. Berglund, H. D. Junghenn and P. Milnes, Analysis on semigroups, John Wiley \& Sons. MR 91b:43001

[4] R. E. Bruck, On the almost-convergence of iterates of a nonexpansive mapping in Hilbert space and the structure of the weak $\omega$-limit set, Israel J. Math. 29 (1978), 1-16. MR 58:2474

[5] _ On the convex approximation property and the asymptotic behavior of nonlinear contractions in Banach spaces, Israel J. Math. 38 (1979), 304-314. MR 82h:47051

[6] A. H. Clifford and G. B. Preston, The algebraic theory of semigroups, Vol. 1, American Mathematical Society, 1961. MR 24:A2627 
[7] M. M. Day, Amenable semigroups, Illinois J. Math. 1 (1957), 509-544. MR 19:1067c

[8] - Fixed point theorem for compact convex sets, Illinois J. Math. 5 (1961), 585-590. MR 25:1547

[9] W. F. Eberlein, Abstract ergodic theorems and weak almost periodic functions, Trans. Amer. Math. Soc. 67 (1949), 217-240. MR 12:112a

[10] S. Goldberg and P. Irwin, Weakly almost periodic vector-valued functions, Diss. Math. 157 (1979), 46 pp. MR 80e:43010

[11] N. Hirano, K. Kido and W. Takahashi, Asymptotic behavior of commutative semigroups of nonexpansive mappings in Banach spaces, Nonlinear Analysis 10 (1986), 229-249. MR 87d:47078

[12] Nonexpansive retractions and nonlinear ergodic theorems in Banach spaces, Nonlinear Analysis 12 (1988), 1269-1281. MR 90a:47138

[13] O. Kada, Strong ergodic theorems for commutative semigroups of operators, (submitted).

[14] _ Ergodic theorems for noncommutative semigroups of operators, preprint.

[15] _ Strong ergodic theorems for noncommutative semigroups in Banach spaces, preprint.

[16] O. Kada, A. Lau and W. Takahashi, Asymptotically invariant net and fixed point set for semigroup of nonexpansive mappings, Nonlinear Anal. 29 (1997), 539-550. MR 98d:47156

[17] O. Kada and W. Takahashi, Nonlinear ergodic theorems for almost nonexpansive curves over commutative semigroups, Topological Methods in Nonlinear Analysis 5 (1995), 305-324. MR 97f: 47053

[18] - Strong convergence and nonlinear ergodic theorems for commutative semigroups of nonexpansive mappings, Nonlinear Analysis 28 (1997), 495-511. MR 97j:47075

[19] J. L. Kelley and I. Namioka, Linear topological spaces, Van Nostrand, 1963. MR 29:3851

[20] K. Kido and W. Takahashi, Mean ergodic theorems for semigroups of linear operators, J. Math. Anal. Appl. 103 (1984), 387-394. MR 86g:47052

[21] A. T. Lau and W. Takahashi, Invariant means and fixed point properties for nonexpansive representations of topological semigroups, Topological Methods in Nonlinear Analysis $\mathbf{5}$ (1995), 39-57. MR 96i:47101

[22] P. Milnes, On vector-valued weakly almost periodic functions, J. London Math. Soc. (2) 22 (1980), 467-472. MR 82i:43007

[23] I. Namioka, Følner's condition for amenable semigroups, Math. Scand. 15 (1964), 18-28. MR 31:5062

[24] W. M. Ruess and W. H. Summers, Weak almost periodicity and the strong ergodic limit theorem for contraction semigroups, Israel J. Math. 64 (1988), 139-157. MR 90c:47118

[25] _ Integration of asymptotically almost periodic functions and weak asymptotic almost periodicity, Diss. Math. 279 (1989). MR 90d:46056

[26] _ Weakly almost periodic semigroups of operators, Pacific J. Math. 143 (1990), 175193. MR 91b:47142

[27] _ Ergodic theorems for semigroups of operators, Proc. Amer. Math. Soc. 114 (1992), 423-432. MR 92e:47016

[28] H. H. Schaefer, Topological vector spaces, Springer-Verlag, 1971. MR 49:7722

[29] W. Takahashi, A nonlinear ergodic theorem for an amenable semigroup of nonexpansive mappings in a Hilbert space, Proc. Amer. Math. Soc. 81 (1981), 253-256. MR 82f:47079

[30] _ Nonlinear Functional Analysis (Japanese), Kindai-kagakusha, Japan, 1988.

Department of Information Sciences, Tokyo Institute of Technology, Oh-Okayama, MEguRO-KU, TOKYO 152, JAPAN

E-mail address: kada@math.sci.ynu.ac.jp 